A C G

Rec. Nat. Prod. 13:4 (2019) 287-295

records of natural

publications

products

\title{
A New Diterpene: Abietane Glycoside from the Roots of Isodon rugosus Wall Ex Benth.
}

\section{Arif Ullah ${ }^{1}$, Ghias Uddin $\odot^{1}$, Mamoon Ur Rashid $\odot^{2^{*}}$, Ismail Ismail $\odot^{1}$, Nasrudddin Nasruddin $\odot^{1}$, Bina Shaheen Siddiqui $\odot^{3}$, Zahoor Ullah $\odot^{4}$ and Muhammad Alamzeb ${ }^{5}$}

\author{
${ }^{1}$ Phytopharmaceutical and Nutraceutical Research Laboratoires (PNRL), Institute of Chemical \\ Sciences, University of Peshawar, Peshawar-25120, Pakistan \\ ${ }^{2}$ Ton Duc Thang University (TDTU), Ho Chi Minh City, Vietnam \\ ${ }^{3}$ H.E.J. Research Institute of Chemistry University of Karachi, Karachi, Pakistan \\ ${ }^{4}$ Department of Chemistry, Balochistan University of Information Technology, Engineering and \\ Management Sciences, Quetta-87100, Pakistan \\ ${ }^{5}$ Department of Chemistry, University of Kotli, Kotli-11100, Azad Jammu and Kashmir, Pakistan
}

(Received June 21, 2018; Revised October 08, 2018; Accepted October 10,2018)

\begin{abstract}
The methanolic extract of the roots of Isodon rugosus were subjected to chromatographic separation, yielded two diterpenes: a new compound named rugosodon 1 and a known compound oridonin 2 . The new compound 1 was elucidated as 1-O- $\alpha-D$-glucopyranosyl-7 $\alpha, 13 \beta$-dihydroxyabieta-8(9)-en-11-one and the known compound 2 as 7 2,20 -epoxy-1 $\alpha, 6 \beta, 7,14$-tetrahydroxy-kaur-16-en-15-one (oridonin), based on the chemical hydrolysis, physicochemical and spectroscopic data of IR, ESIMS, EIMS, 1D, and 2D NMR. The compounds 1$\mathbf{2}$ were subjected to bioassay studies of cytotoxicity and $\alpha$-glucosidase inhibition potential. Rugosodon 1 showed significant potential of $\alpha$-glucosidase inhibition with $\mathrm{IC}_{50} 0.453 \mathrm{mg} / \mathrm{mL}$, as compared to the standard acarbose $\left(\mathrm{IC}_{50} 0.921 \mathrm{mg} / \mathrm{mL}\right)$. The compounds $\mathbf{1 - 2}$ failed to show any significant results for the cytotoxic screening against the three human cancer cell lines (NCI-H460, HeLa and MCF-7).
\end{abstract}

Keywords: Abietane; diterpene; glycoside; Isodon rugosus; rugosodon; oridonin. (C) 2019 ACG Publications. All rights reserved.

\section{Introduction}

Isodon, formally known as Rabdosia of Labiatae (Lamiaceae) is a medicinally important genus consists of approximately 150 species. It is a cosmopolitan genus, distributed almost everywhere in the world, however, it is predominantly originated in Asia's tropical and subtropical regions [1]. Isodon rugosus Wall. ex Benth (Lamiaceae) is found in the northern Pakistan where it is locally known as phaypush, boi and sperkai [2-3]. The plant is traditionally used as antiseptic, hypoglycaemic, antidiarrheal, bronchodilator and instant anti-scabies [4-7]. The extracts from aerial parts (leaves and flowers) are particularly used for the treatment of hypertension, toothache, fevers and rheumatism. Recently, the phytochemical analysis of I. rugosus have reported six cytotoxic diterpenes [6, 8].

\footnotetext{
*Corresponding author: E-Mail: mamoon.urrashid@tdtu.edu.vn Phone:+923339688051 
Various types of bioactive diterpenes including ent-halimane, ent-clerodane, ent-labdane, abietane, ent-abietane, ent-pimarane, ent-totarane and Nor, Seco, monomeric, and dimeric ent-kaurane are reported from different Isodon species [4, 5, 9-11]. The abietane class has structure similarity with the chinane, sempervirane, and totarane types of diterpenes which are classified based on the position of isopropyl group at $\mathrm{C}-13, \mathrm{C}-11, \mathrm{C}-12$, and $\mathrm{C}-14$, respectively [12-16]. Here, we are reporting the isolation, structural identification, and bioactivities (cytotoxicity, and $\alpha$-glucosidase inhibitory potential) of the compounds 1-2 (Figure 1) obtained from the roots of I. rugosus Wall Ex. Benth.

\section{Materials and Methods}

\subsection{General Experimental Procedures}

Bicote melting point apparatus (Bibby Scientific limited, UK) via glass capillaries tubes was used for melting points determination. UNICAM UV 300 and FT/IR-4200 (ATR, in solid state) spectrophotometer were used for recording Ultraviolet (UV) and Infrared spectra (IR), respectively. AA-100 polarimeter with sodium lamp (D-line, $589.3 \mathrm{~nm}$ ) at $20^{\circ} \mathrm{C}$ (Perkin Elmer, USA) was used for measuring the optical rotations $[\alpha]_{D}^{20}$, in methanol. JEOL MS 600H-1 (Japan) and Q-TOF/6500 series (Germany) mass spectrometer were used for recording the EIMS and ESIMS spectra, respectively. The ID-NMR including ${ }^{1} \mathrm{H}(400 \mathrm{MHz}),{ }^{13} \mathrm{C}(150 \mathrm{MHz})$, and 2D NMR (COSY, NOESY, HSQC, HMBC) spectra were recorded in methanol d4, TMS (tetramethylsilane) as internal standard with Bruker (Avance DRX-400 and $600 \mathrm{MHz}$ ) NMR spectrophotometers. The chemical shifts $(\delta)$ and coupling constant $(J)$ values for ${ }^{1} \mathrm{H}$ and ${ }^{13} \mathrm{C}$ are recorded in ppm and $\mathrm{Hz}$, respectively. The column chromatography was performed using silica gel ( $\mathrm{Si}$ 60, 80-230 mesh) and prep. TLC through aluminum sheets $(0.2 \mathrm{~mm}$ thickness) precoated with silica gel 60PF254 (Merck), respectively. The concentration of $p$-nitrophenol was measured at $405 \mathrm{~nm}$ using Epoch 2 Multiplate Reader (BioTek Instruments, Inc., USA). Metabolites spots on the TLC plates were visualized by examining under UV light and spraying with $\mathrm{Ce}\left(\mathrm{SO}_{4}\right)_{2}$ solution followed by heating.

\subsection{Collection and Identification of Plant Materials}

The whole plant of I. rugosus (roots, stems, leaves and flowers) were collected from Bajaur Agency (FATA), Pakistan in August 2014 and identified by Dr. Ghulam Jelani, (plant taxonomist). The plant sample with voucher No. 4566-2016 was deposited at the herbarium, Department of Botany, University of Peshawar, Pakistan.

\subsection{Extraction and Isolation of Compounds 1-2}

The shadow dried roots of $I$. rugosus $(6.0 \mathrm{Kg})$ were powdered by blender, soaked in $(9.0 \mathrm{~L})$ methanol for twelve days and extracted repeatedly $(4 \mathrm{x})$, with methanol (recycled), at room temperature. It was followed by rotary evaporation yielded a dark red residue $(600 \mathrm{~g})$. The residue obtained was suspended in water and subsequently extracted with n-hexane, $\mathrm{CHCl}_{3}$ and EtOAc, yielded four corresponding solvents fractions (including the aqueous fraction), respectively. A slurry was made by absorbing the $\mathrm{CHCl}_{3}$ fraction $(200 \mathrm{~g})$ on silica gel $(200 \mathrm{~g})$, and then subjected to column chromatography (CC) (prepacked silica gel column, $1.2 \mathrm{~kg}$ ), using solvent system (n-hexane - EtOAc, $1: 0,100: 1,75: 1,50: 1,25: 1,5: 1)$ yielded six fractions $(\mathrm{J}-\mathrm{O})$. Fraction $\mathrm{L}(25.0 \mathrm{~g})$ on $\mathrm{CC}$ (silica gel, $400 \mathrm{~g}$ ) using solvent system (n-hexane - EtOAc, 10: 1, 5: 1, 2:1, 0:1) yielded four subfractions L1, L2, L3 and L4. Sub-fraction L2 (10 g) on CC (silica gel, $160 \mathrm{~g}$ ), followed by preparative TLC using solvent system (n-hexane - EtOAc, $50: 50$ ), furnished compound 1 (rugosodon, $15.0 \mathrm{mg}$ ). Fraction M (54 g) on CC (silica gel, $800 \mathrm{~g}$ ) using solvent system (n-hexane - EtOAc, $5: 1,2: 1,1: 1$ ) furnished three sub-fractions M1, M2 and M3. Sub-fraction M2 (15 g) was subjected to prep. TLC using solvent system (n-hexane - EtOAc, $60: 40$ ) and furnished compound 2 (oridonin, $9.5 \mathrm{mg}$ ). 


\section{4. $\alpha$-Glucosidase Inhibitory Activity}

To perform the assay of $\alpha$-glucosidase inhibition by rugosodon $\mathbf{1}$ and oridonin $\mathbf{2}$ in the presence of Acarbose as standard was performed in a 96 well plate per the standard protocol with minor modification [21]. The preincubation at $37^{\circ} \mathrm{C}(15 \mathrm{~min})$ was performed by adding $20 \mu \mathrm{L}$ of samples (rugosodon 1 and oridonin 2 and standard) at varying concentrations $(0.1,0.2,0.3,0.4$, and 0.5 $\mathrm{mg} / \mathrm{mL})$ to a mixture of $10 \mu \mathrm{L} \alpha$-glucosidase $(1 \mathrm{U} / \mathrm{ml})$ in $50 \mu \mathrm{L}$ of phosphate buffer $(100 \mathrm{mM}, \mathrm{pH}=6$. 8) It was followed by the addition of $20 \mu \mathrm{L}$ P-NPG $(5 \mathrm{mM})$ as a substrate and further incubation at $37^{\circ} \mathrm{C}(20 \mathrm{~min})$. Finally, to terminate the reaction, a $50 \mu \mathrm{L} \mathrm{Na}_{2} \mathrm{CO}_{3}(0.1 \mathrm{M})$ was added to the reaction mixture. Multiplate Reader was used to detect the release of $p$-nitrophenol by measuring its absorbance at $405 \mathrm{~nm}$. Each experiment was performed in triplicate before the test substance was set up in parallel as a control. The data is presented as means values obtained through \pm standard deviation and $\mathrm{IC}_{50}$ values were calculated using GraphPad Prism 5.01 (La Jolla, CA, USA.). The following formula was used to calculate the results expressing $\%$ inhibition.

$\%$ Inhibitory $=(1-\mathrm{As} / \mathrm{Ac}) \times 100$. In this equation, As and Ac is the absorbance of the control with and without adding the test substance, respectively.

\subsection{Sulforhodamine B (SRB) Colorimetric Assay for Cytotoxicity Screening}

The cytotoxicity (in vitro) assay is widely used to identify the concentration of a drug sufficient to inhibit cell growth and initiate program cell death [22]. By using EDTA-trypsin, $0.02 \%$ : $0.05 \%$, the monolayer of the three cells types was trypsinised for 2-3 min yielded cell suspension. The concentration of each cell line and cell viability was determined by using hemocytometer. The proper cell counts $(2 \times 104)$ of cell suspension $(100 \mathrm{~mL} /$ well $)$ of each cell types NCI-H460, MCF-7 and $\mathrm{HeLa}$, was added in each well of 96-well plates except the blank and incubated (for $24 \mathrm{~h}$ ) in $5 \% \mathrm{CO}_{2}$ atmosphere, at $37^{\circ} \mathrm{C}$. A $100 \mathrm{~mL}$ of the test samples: rugosodon 1, and oridonin $2(0.001-100 \mathrm{mM})$ and the standard anticancer drugs cisplatin $(0.1-100 \mathrm{mM})$ and doxorubicin $(0.001-10 \mathrm{mM})$ were transferred in their selected wells. The plates were incubated at $37^{\circ} \mathrm{C}(48 \mathrm{~h})$, followed by ice-cold TCA $(50 \mathrm{~mL}, 50 \%)$ fixation $(30 \mathrm{~min})$ and finally washed with distilled water. The plates after the overnight air-dryness were stained with SRB $(0.4 \%)$ for $30 \mathrm{~min}$. Acetic acid $(1 \%)$ was used to remove the unbound stain. The SRB stain was dissolved using Tris-base $(100 \mathrm{~mL}, 10 \mathrm{mM})$ followed by spectrophotometry measurement of the optical density at $515 \mathrm{~nm}$. The $\mathrm{LC}_{50}\left(50 \%\right.$ cell death) and $\mathrm{GI}_{50}$ (50\% growth inhibition) which represent the vital concentration of a sample enough for causing 50\% cell death and growth inhibition of total cells, respectively, were obtained graphically as concentration $v s$ cytotoxicity and \% inhibition of cell growth.

\subsection{Acid Hydrolysis of Rugosodon (1)}

Rugosodon 1 ( $2 \mathrm{mg}$ in methanol) and $\mathrm{HCl}\left(5 \mathrm{~mL}, 2 \mathrm{~N}\right.$ aq.) were mixed and refluxed at $100^{\circ} \mathrm{C}$, for $4 \mathrm{~h}$. $\mathrm{CHCl}_{3}(3 \times 5 \mathrm{~mL})$ was used to selectively extract the aglycone part from the reaction mixture. A saturated solution of $\mathrm{Na}_{2} \mathrm{CO}_{3}$ was used to neutralize the residual aqueous fraction (containing sugar) and then subjected to TLC analysis in the presence of a standard D-glucose sample (using the solvent system MeCOEt-isoPrOH- Me2CO-H2O 20:10:7:6). The values of the retention factor were compared (Rf: 0.40), and the sugar was identified as D-glucose [18, 20]. 

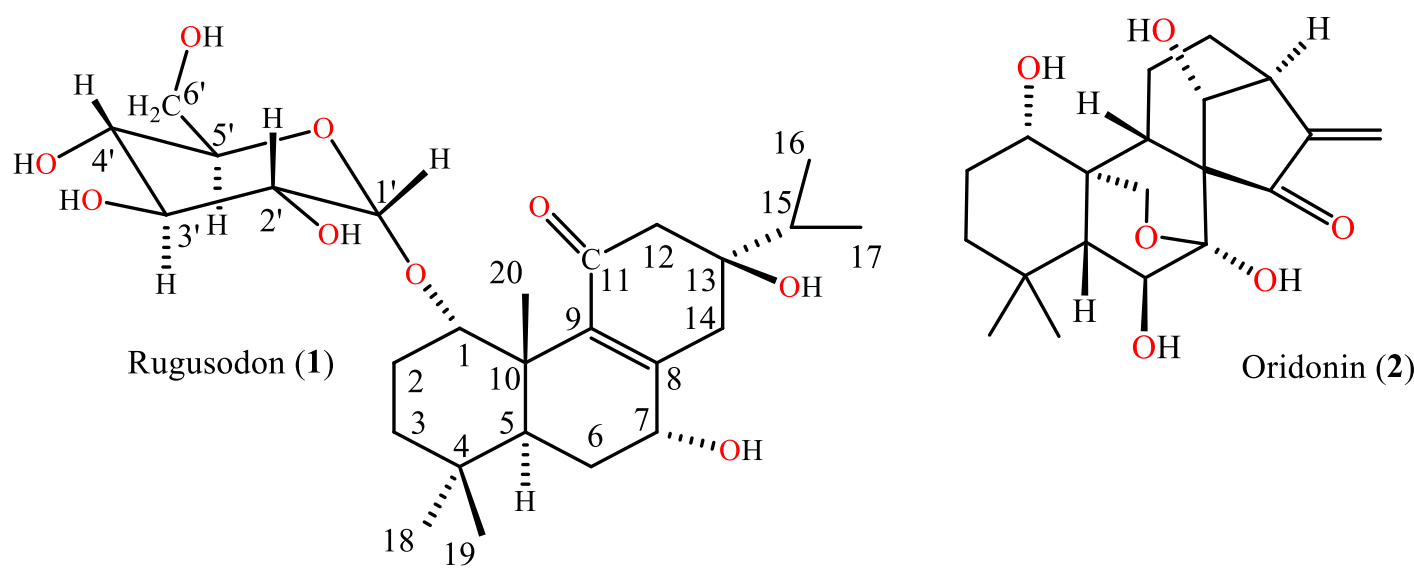

Figure 1. Structures of rugosodon (1) and oridonin (2)

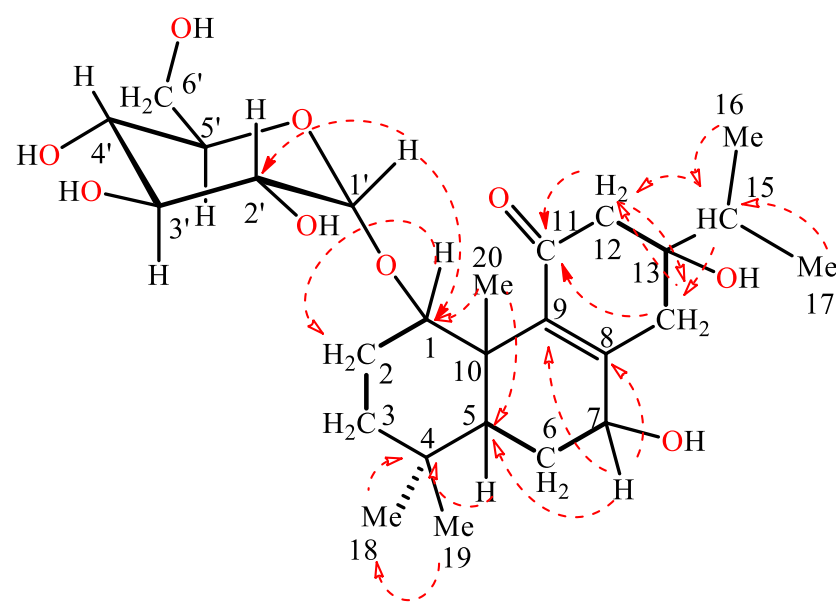

Figure 2. Selected COSY $(\longrightarrow)$ and $\operatorname{HMBC}(\cdots \Delta)$ of rugosodon $(\mathbf{1})$

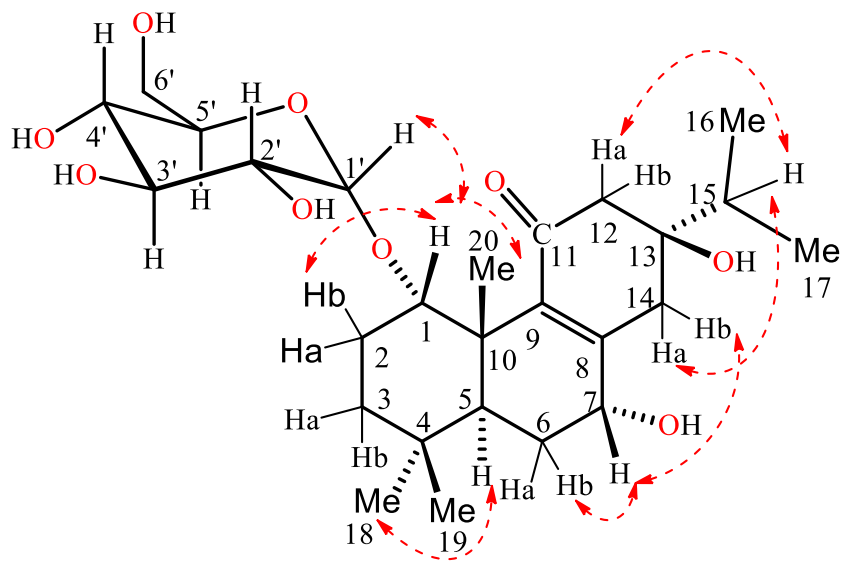

Figure 3. Selected NOESY correlations ( 


\section{Results and Discussion}

\subsection{Structure Elucidation}

Rugosodon $1(\mathrm{MeOH})$ (Figure 1) was obtained as a white amorphous powder, possessed a molecular formula of $\mathrm{C}_{26} \mathrm{H}_{42} \mathrm{O}_{9}$ based on the pseudo molecular ion peak in the positive ion ESIMS at $m / z: 521.2727[\mathrm{M}+\mathrm{Na}]^{+}$(cald. $m / z: 521.2723$ for $\mathrm{C}_{26} \mathrm{H}_{42} \mathrm{NaO}_{9}$ ) and ${ }^{13} \mathrm{C}$ NMR data (Figures S1-S4 and S7-12, supplementary materials). The IR spectra of $\mathbf{1}$ indicate the presence of hydroxyl groups (3490-3260), carbonyl (1712) and olefinic carbon $\left(1640 \mathrm{~cm}^{-1}\right)$. The compound 1 showed optical activity: $[\alpha]_{D}^{20}=+55.89$ (c 0.07 , methanol) and UV $(\mathrm{MeOH}) \lambda_{\max }: 239 \mathrm{~nm}$. The NMR spectra of compound 1 showed similarities to the reference compound 'Isoabietenin B' [17] isolated from $I$. tenuifolius except for the additional sugar moeity at $\mathrm{C}-1$ of rugosodon 1 while a third hydroxyl group is present at $\mathrm{C}-18$ of Isoabietenin B. The analysis of the ${ }^{13} \mathrm{C}$ NMR spectra (BB) showed 26 carbons differentiated into four $\mathrm{sp}^{3}$ and two $\mathrm{sp}^{2}$ quaternary carbons, nine $\mathrm{sp}^{3}$ methines, six $\mathrm{sp}^{3}$ methylenes and five methyls (Table 1, supplementary materials), suggested glucose substituted tricyclic diterpene skeleton for compound $\mathbf{1}$.

The ${ }^{1} \mathrm{H}$ NMR signals (Table S3-S5, supplementary materials) and HMBC correlations traced from five methyls $\left[\mathrm{H}_{3}-16 / \mathrm{H}_{3}-17\left(\delta_{\mathrm{H}} 0.92 \mathrm{~d}, 6.8 \mathrm{~Hz} / 0.93 \mathrm{~d}, 6.8 \mathrm{~Hz}\right)\right.$ to $\mathrm{C}-15\left(\delta_{\mathrm{C}} 39.54\right), \mathrm{C}-13\left(\delta_{\mathrm{C}}\right.$ 77.61); $\mathrm{H}_{3}-19\left(\delta_{\mathrm{H}} 0.89\right)$ and $\mathrm{H}_{3}-18\left(\delta_{\mathrm{H}} 0.94\right)$ to $\mathrm{C}-4\left(\delta_{\mathrm{C}} 33.42\right)$ and $\mathrm{H}_{3}-20\left(\delta_{\mathrm{H}} 1.18\right)$ to $\left.\mathrm{C}-10\left(\delta_{\mathrm{C}} 44.66\right)\right]$ along with the COSY correlation of $\mathrm{H}_{3}-16 / \mathrm{H}_{3}-17$ with $\mathrm{H}-15$ suggested compound $\mathbf{1}$ to be an abietane diterpenoid [17]. The characteristic HMBC correlations from the anomeric oxymethine $\mathrm{H}-1^{\prime}\left(\delta_{\mathrm{H}} 3.98\right.$, $\mathrm{d} 7.6 \mathrm{~Hz})$ of the glucose moiety to the glycosylated methine $\mathrm{C}-1\left(\delta_{\mathrm{C}} 80.91\right)$ as well as the characteristic ${ }^{1} \mathrm{H} /{ }^{13} \mathrm{C}$ NMR signals of the glucose moiety comprising four oxymethines at $\left[\mathrm{H}-2^{\prime}\left(\delta_{\mathrm{H}} 3.10\right.\right.$, dd $2.8,7.6$ $\left.\mathrm{Hz} ; \delta_{\mathrm{C}} 75.10\right), \mathrm{H}-3^{\prime}\left(\delta_{\mathrm{H}} 3.24\right.$, dd $\left.2.8,2.4 \mathrm{~Hz} ; \delta_{\mathrm{C}} 77.76\right), \mathrm{H}-4^{\prime}\left(\delta_{\mathrm{H}} 3.25\right.$, overlap; $\left.\delta_{\mathrm{C}} 71.87\right), \mathrm{H}-5^{\prime}\left(\delta_{\mathrm{H}} 3.16\right.$, ddd 5.6, 3.2, $\left.\left.2.4 \mathrm{~Hz} ; \delta_{\mathrm{C}} 77.71\right)\right]$ and oxymethylene at $\left[\mathrm{H} \alpha-6^{\prime}\left(\delta_{\mathrm{H}} 3.80\right.\right.$, dd 11.6, $\left.2.4 \mathrm{~Hz} ; \delta_{\mathrm{C}} 62.86\right)$ and $\mathrm{H} \beta-6^{\prime}\left(\delta_{\mathrm{H}} 3.64\right.$, dd $\left.\left.11.6,5.6 \mathrm{~Hz}\right)\right]$ proposed that $\mathrm{C}-1$ of the abietane system is substituted with glucose moiety.

The position of the carbonyl at $\mathrm{C}-11\left(\delta_{\mathrm{C}} 204.08\right)$, tetra-substituted double bond $\left(\Delta^{8,9}\right)$ at $\mathrm{C}-8$ $\left(\delta_{\mathrm{C}} 151.05\right)$ and $\mathrm{C}-9\left(\delta_{\mathrm{C}} 141.44\right)$ and the two hydroxyl groups at C-7 $\left(\delta_{\mathrm{C}} 70.75\right)$ and $\mathrm{C}-13\left(\delta_{\mathrm{C}} 77.61\right)$, respectively, were traced from the characteristics ${ }^{13} \mathrm{C}-\mathrm{NMR}$ signals and $\mathrm{HMBC}$ correlation from $\mathrm{H}-7$ $\left(\delta_{\mathrm{H}} 4.05\right.$, dd $\left.2.8,2.4 \mathrm{~Hz}\right)$ to $\mathrm{C}-5\left(\delta_{\mathrm{C}} 40.29\right), \mathrm{C}-8$, and C-9; from $\mathrm{H}_{2} \alpha / \beta-12\left(\delta_{\mathrm{H}} 2.99 \mathrm{~d}, 14.4 \mathrm{~Hz} / 2.28 \mathrm{dd}\right.$, $14.4,2.0 \mathrm{~Hz})$ to $\mathrm{C}-9, \mathrm{C}-11$ (carbonyl), $\mathrm{C}-13$, and C-14 $\left(\delta_{\mathrm{C}} 40.13\right)$; from $\mathrm{H}_{2} \alpha / \beta-14\left(\delta_{\mathrm{H}} 2.80 \mathrm{~d}, 18.0 \mathrm{~Hz} /\right.$ $2.19 \mathrm{dd}, 18.0,2.0 \mathrm{~Hz})$ to $\mathrm{C}-7, \mathrm{C}-8, \mathrm{C}-9, \mathrm{C}-13$, and $\mathrm{C}-15(39.54)$; and from $\mathrm{H}_{2} \alpha / \beta-6\left(\delta_{\mathrm{H}} 1.82\right.$, overlap/ 1.76, overlap) to C-5 ( $\left.\delta_{\mathrm{C}} 40.29\right)$, and C-7, respectively. Furthermore, the COSY correlation between $\mathrm{H}_{2} \alpha / \beta-2\left(\delta_{\mathrm{H}} 2.03,14.2,2.8 \mathrm{~Hz} / 1.88\right.$, overlap) with methylenes $\mathrm{H}_{2} \alpha / \beta-3\left(\delta_{\mathrm{H}} 1.76\right.$, overlap/1.14, overlap) and glycosylated-methine $\mathrm{H}-1\left(\delta_{\mathrm{H}} 4.56, \mathrm{dd} 2.2,2.8 \mathrm{~Hz}\right)$; and $\mathrm{H}_{2} \alpha / \beta-6\left(\delta_{\mathrm{H}} 1.82\right.$, overlap/ 1.76, overlap) with methine $\mathrm{H}-5\left(\delta_{\mathrm{H}} 1.93\right.$, dd $\left.6.4,3.2 \mathrm{~Hz}\right)$ and oxymethine $\mathrm{H}-7\left(\delta_{\mathrm{H}} 4.05\right.$, dd $\left.2.8,2.4 \mathrm{~Hz}\right)$ establish the structure of compound $\mathbf{1}$ as 1- $O-D$-glucopyranosyl-7,13-dihydroxyabieta-8(9)-en-11-one.

To further confirm the structure of sugar moiety obtained after acid hydrolysis $(5 \% \mathrm{HCl})$ of compound 1 was confirmed as $D$-glucose by comparing its retention factor ( $R f: 0.40)$ value on TLC $\left(\mathrm{MeCOEt}-\mathrm{Me}_{2} \mathrm{CO}-\mathrm{isoPrOH}-\mathrm{H}_{2} \mathrm{O}, 20: 7: 10: 6\right)$ and specific rotation $=+52.19(c=0.1$ in water $)$ with the standard D glucose sample $[18,21]$. The relative stereochemistry of compound $\mathbf{1}$ was assigned from the selected NOESY correlations of H-7/ H-14 $\beta$, H-6 $\beta ; \mathrm{H}-1 / \mathrm{H}-1^{\prime}, \mathrm{H}-2 \beta, \mathrm{H}_{3}-20 ; \mathrm{H}-5 /$ $\mathrm{H}_{3}-18$; and $\mathrm{H}-12 \alpha$ and $\mathrm{H}-14 \alpha / \mathrm{H}-15$ indicated the relative configurations of $\mathrm{H}-1, \mathrm{H}-7$ and $\mathrm{HO}-13$ as $\beta$ oriented.

These evidences assign the relative configuration and structure assignment of compound $\mathbf{1}$ as $(1 S, 5 S, 7 R, 10 S, 13 S)-1-\mathrm{O}-\alpha-D$-glucopyranosyl-7 $\alpha, 13 \beta$-dihydroxyabieta-8(9)-en-11-one.

The selected COSY (Images S13-S17) and HMBC (Images S23-S26) correlation of compound 1 are given in Figure 2 and NOESY correlation in Figure 3, respectively (supplementary material).

The compound 2 (Figure 1) was isolated as a white crystal. From the ${ }^{1} \mathrm{H}$ NMR and ${ }^{13} \mathrm{C}$ NMR (Table 2) data, it was identified as a known compound oridonin $(7 \alpha, 20$-epoxy-1 $\alpha, 6 \beta, 7,14-$ tetrahydroxy-kaur-16-en-15-one) [19]. 
Table 1. ${ }^{1} \mathrm{H}\left(\mathrm{CD}_{3} \mathrm{OD}, 400 \mathrm{MHz}\right),{ }^{13} \mathrm{C}$ NMR $\left(\mathrm{CD}_{3} \mathrm{OD}, 150 \mathrm{MHZ}\right), \mathrm{COSY}\left({ }^{1} \mathrm{H} \leftrightarrow{ }^{1} \mathrm{H}\right),\left(\mathrm{CD}_{3} \mathrm{OD}, 600\right.$ $\mathrm{MHz})$ and $\mathrm{HMBC}\left[{ }^{1} \mathrm{H}(600 \mathrm{MHz}) \rightarrow{ }^{13} \mathrm{C}(150 \mathrm{MHz}), \mathrm{CD}_{3} \mathrm{OD}\right]$ of Compound 1 (Rugosodon)

\begin{tabular}{|c|c|c|c|c|}
\hline Position & $\delta^{1} \mathbf{H}$ & $\delta^{13} \mathrm{C}$ & $\operatorname{COSY}\left({ }^{1} \mathrm{H} \leftrightarrow{ }^{1} \mathbf{H}\right)$ & HMBC $\left({ }^{1} \mathbf{H} \rightarrow{ }^{13} \mathbf{C}\right)$ \\
\hline 1 & $4.56, \mathrm{dd}(2.2,2.8)$ & 80.91 & $\mathrm{H}-1 \leftrightarrow \mathrm{H} \alpha / \beta-2$ & $\mathrm{H}-1 \rightarrow \mathrm{C}-3, \mathrm{C}-10$ \\
\hline $2 \alpha$ & 1.88 , overlap & 25.52 & $\mathrm{CH}_{2} \alpha / \beta-2 \leftrightarrow \mathrm{H} \beta-2, \mathrm{H} \alpha / \beta-3, \mathrm{H}-$ & $\mathrm{H} \alpha / \beta-2 \rightarrow \mathrm{C}-3, \mathrm{C}-20$ \\
\hline $2 \beta$ & $2.03, \mathrm{dd}(14.2,2.8)$ & & 1 & \\
\hline $3 \alpha$ & 1.76 , overlap & 36.19 & $\mathrm{H} \alpha / \beta-3 \leftrightarrow \mathrm{H} \alpha / \beta-2$ & $\mathrm{H} \alpha / \beta-3 \rightarrow \mathrm{C}-2, \mathrm{C}-10$ \\
\hline $3 \beta$ & 1.14 , overlap & & & \\
\hline 4 & --- & 33.42 & --- & --- \\
\hline 5 & $1.93, \mathrm{dd}(6.4,3.2)$ & 40.29 & $\mathrm{H}-5 \leftrightarrow \mathrm{H} \alpha / \beta-6$ & $\begin{array}{l}\mathrm{H}-5 \rightarrow \mathrm{C}-4, \mathrm{C}-6, \mathrm{C}-7, \mathrm{C}-10, \\
\mathrm{C}-18, \mathrm{C}-20\end{array}$ \\
\hline $6 \alpha$ & 1.82 , overlap & 28.59 & $\mathrm{H} \alpha / \beta-6 \leftrightarrow \mathrm{H}-5, \mathrm{H}-7$ & $\mathrm{H} \alpha / \beta-6 \rightarrow \mathrm{C}-7, \mathrm{C}-10$ \\
\hline $6 \beta$ & 1.76 , overlap & & & \\
\hline 7 & $4.05, \mathrm{dd}(2.8,2.4)$ & 70.75 & $\mathrm{H}-7 \leftrightarrow \mathrm{H} \alpha / \beta-6$ & $\mathrm{H}-7 \rightarrow \mathrm{C}-5, \mathrm{C}-8, \mathrm{C}-9$ \\
\hline 8 & --- & 151.05 & --- & --- \\
\hline 9 & --- & 141.44 & --- & --- \\
\hline 10 & --- & 44.66 & --- & --- \\
\hline 11 & --- & 204.08 & --- & --- \\
\hline $\begin{array}{l}12 \alpha \\
12 \beta\end{array}$ & $\begin{array}{l}2.99, \mathrm{~d}(14.4) \\
2.28, \mathrm{dd}(14.4,2.0)\end{array}$ & 52.80 & $\begin{array}{l}\mathrm{H} \alpha-12 \leftrightarrow \mathrm{H} \beta-12) \text { and } \mathrm{H} \beta-12 \leftrightarrow \\
\mathrm{H} \alpha-12, \mathrm{H} \beta-14\end{array}$ & $\begin{array}{l}\mathrm{H} \alpha-12 \rightarrow \mathrm{C}-11, \mathrm{C}-13 \text { and } \mathrm{H} \beta \\
12 \rightarrow \mathrm{C}-9, \mathrm{C}-11, \mathrm{C}-13, \mathrm{C}-14\end{array}$ \\
\hline 13 & --- & 77.61 & --- & --- \\
\hline $\begin{array}{l}14 \alpha \\
14 \beta\end{array}$ & $\begin{array}{l}2.80, \mathrm{~d}(18) \\
2.19, \mathrm{dd}(18,2.0)\end{array}$ & 40.13 & $\begin{array}{l}\mathrm{H} \alpha-14 \leftrightarrow \mathrm{H} \beta-14) \text { and } \mathrm{H} \beta- \\
14 \leftrightarrow \mathrm{H} \alpha-14, \mathrm{H} \beta-12\end{array}$ & $\begin{array}{l}\mathrm{H} \alpha-14 \rightarrow \mathrm{C}-8, \mathrm{C}-9, \mathrm{C}-11, \mathrm{C}- \\
13, \mathrm{C}-15 \text { and } \\
\mathrm{H} \beta-14 \rightarrow \mathrm{C}-7, \mathrm{C}-8, \mathrm{C}-9, \mathrm{C}- \\
11, \mathrm{C}-12, \mathrm{C}-13\end{array}$ \\
\hline 15 & 1.66, sept $(6.8)$ & 39.54 & $\mathrm{H}-15 \leftrightarrow 3 \mathrm{H}-16,3 \mathrm{H}-17$ & $\mathrm{H}-15 \rightarrow \mathrm{C}-16, \mathrm{C}-17, \mathrm{C}-13$ \\
\hline 16 & $0.92, \mathrm{~d}(6.8)$ & 17.48 & $3 \mathrm{H}-16 \leftrightarrow \mathrm{H}-15$ & $3 \mathrm{H}-16 \rightarrow \mathrm{C}-13, \mathrm{C} 15, \mathrm{C}-17$ \\
\hline 17 & $0.93, \mathrm{~d}(6.8)$ & 16.93 & $3 \mathrm{H}-17 \leftrightarrow \mathrm{H}-15$ & $3 \mathrm{H}-17 \rightarrow \mathrm{C}-13, \mathrm{C} 15, \mathrm{C}-16$ \\
\hline $18 \alpha$ & $0.94, \mathrm{~s}$ & 33.63 & --- & $3 \mathrm{H}-19 \rightarrow \mathrm{C}-4, \mathrm{C}-3, \mathrm{C}-18$ \\
\hline $19 \beta$ & $0.89, \mathrm{~s}$ & 22.13 & --- & $3 \mathrm{H}-18 \rightarrow \mathrm{C}-4, \mathrm{C}-3, \mathrm{C}-19$ \\
\hline 20 & $1.18, \mathrm{~s}$ & 19.59 & --- & $3 \mathrm{H}-20 \rightarrow \mathrm{C}-1, \mathrm{C}-5, \mathrm{C}-9, \mathrm{C}-10$ \\
\hline $1^{\prime}$ & $3.98, \mathrm{~d}(7.6)$ & 105.87 & $\mathrm{H}-1^{\prime} \leftrightarrow \mathrm{H}-2^{\prime}$ & $\mathrm{H}-1^{\prime} \rightarrow \mathrm{C}-1, \mathrm{C}-2^{\prime}, \mathrm{C}-3^{\prime}$ \\
\hline $2^{\prime}$ & $3.10, \mathrm{dd}(7.6,2.8)$ & 75.10 & $\mathrm{H}-2^{\prime} \leftrightarrow \mathrm{H}-3^{\prime} \mathrm{H}-1^{\prime}$ & $\mathrm{H}-2^{\prime} \rightarrow \mathrm{C}-1^{\prime}, \mathrm{C}-3^{\prime}$ \\
\hline $3^{\prime}$ & $3.24, \mathrm{dd}(2.8,2.4)$ & 77.76 & $\mathrm{H}-3^{\prime} \leftrightarrow \mathrm{H}-2^{\prime}, \mathrm{H}-4^{\prime}$ & $\mathrm{H}-3^{\prime} \rightarrow \mathrm{C}-2^{\prime}, \mathrm{C}-4^{\prime}$ \\
\hline $4^{\prime}$ & 3.25 , overlap & 71.87 & $\mathrm{H}-4^{\prime} \leftrightarrow \mathrm{H}-5^{\prime}, \mathrm{H}-3^{\prime}$ & $\mathrm{H}-4^{\prime} \rightarrow \mathrm{C}-3^{\prime}, \mathrm{C}-5^{\prime}$ \\
\hline $5^{\prime}$ & $\begin{array}{l}3.16 \text {, ddd (5.6, 3.2, } \\
2.4)\end{array}$ & 77.71 & $\mathrm{H}-5^{\prime} \leftrightarrow \mathrm{H}-4^{\prime}, \mathrm{H} \alpha / \beta-6^{\prime}$ & $\mathrm{H}-5^{\prime} \rightarrow \mathrm{C}-6^{\prime}, \mathrm{C}-4^{\prime}$ \\
\hline $\begin{array}{l}6^{\prime} \alpha \\
6^{\prime} \beta\end{array}$ & $\begin{array}{l}3.80, \mathrm{dd}(11.6,2.4) \\
3.64 \mathrm{dd}(116.5 .6)\end{array}$ & 62.86 & $\begin{array}{l}\mathrm{H} \alpha-6^{\prime} \leftrightarrow \mathrm{H} \beta-6^{\prime}, \mathrm{H}-5^{\prime} \text { and } \mathrm{H} \beta- \\
6^{\prime} \leftrightarrow \mathrm{H} \alpha-6^{\prime} . \mathrm{H}-5^{\prime}\end{array}$ & $\begin{array}{l}\mathrm{H} \alpha-6^{\prime} \rightarrow \mathrm{C}-5^{\prime} \text { and } \\
\mathrm{H} \beta-6^{\prime} \rightarrow \mathrm{C}-4^{\prime} \text { C- } 1^{\prime}\end{array}$ \\
\hline
\end{tabular}

3.2. Physicochemical and Spectroscopic Data of Rugosodon (1) and Oridonin (2)

3.2.1.(1S,5S,7R,10S,13S)-1-O- $\alpha-D$-glucopyranosyl-7 $\alpha, 13 \beta$-dihydroxyabieta-8(9)-en-11-one (rugosodon 1): White powder; $[\alpha]_{D}^{20}=+55.89$ (c 0.40, MeOH); IR $v_{\max } 3490,2922,1712,1646, \mathrm{~cm}^{-1}$; ESIMS at $m / z: 521.2727\left[\mathrm{M}+\mathrm{Na}^{+}\right.$(cald. for $\mathrm{C}_{26} \mathrm{H}_{42} \mathrm{NaO}_{9}$ ), EIMS $\mathrm{m} / z: 480.3(1.7)\left[\mathrm{M}-\mathrm{H}_{2} \mathrm{O}\right]^{\circ+}$ $\left(\mathrm{C}_{26} \mathrm{H}_{42} \mathrm{O}_{9}-\mathrm{H}_{2} \mathrm{O}\right), 462.3$ (1.3) $\left[\mathrm{M}-2 \mathrm{H}_{2} \mathrm{O}\right]^{\circ+}\left(\mathrm{C}_{26} \mathrm{H}_{42} \mathrm{O}_{9}-2 \mathrm{H}_{2} \mathrm{O}\right), 335.3$ (5.0) $\left[\mathrm{C}_{25} \mathrm{H}_{39} \mathrm{O}_{6}\right]^{0+}, 317.2$ (100) $\left[\mathrm{M}-\mathrm{H}_{2} \mathrm{O}-\mathrm{C}_{6} \mathrm{H}_{12} \mathrm{O}_{5}\right]^{0+}, 300.2(57.6)\left[\mathrm{C}_{20} \mathrm{H}_{28} \mathrm{O}_{2}\right]^{0+}, 282.2(27.0)\left[\mathrm{C}_{20} \mathrm{H}_{28} \mathrm{O}\right]^{0+}, 267.2(26.7)\left[\mathrm{C}_{20} \mathrm{H}_{27}\right]^{0+}, 253.2$ 
(7.0) $\left[\mathrm{C}_{19} \mathrm{H}_{25}\right]^{0+}, 226.2$ (89.5) $\left[\mathrm{C}_{17} \mathrm{H}_{22}\right]^{\circ+}, 213.1$ (19.1) $\left[\mathrm{C}_{16} \mathrm{H}_{21}\right]^{0+}, 159.1$ (11.0) $\left[\mathrm{C}_{12} \mathrm{H}_{15}\right]^{0+}, 121.1$ (7.4), $\left[\mathrm{C}_{9} \mathrm{H}_{13}\right]^{0+}$ (S1, supplementary materials).

${ }^{1} \mathrm{H}$ NMR (CD ${ }_{3} \mathrm{OD}, 400 \mathrm{MHz}$ ) (Table 1): $\delta 4.56(1 \mathrm{H}, \mathrm{d}, J=2.2,2.8 \mathrm{~Hz}, \mathrm{H}-1), 1.88$ (1H, overlap, $\mathrm{H} \alpha-2)$ and $2.03(14.2,2.8 \mathrm{~Hz}, \mathrm{H} \beta-2), 1.76(1 \mathrm{H}$, overlap, $\mathrm{H} \alpha-3)$ and $1.14(1 \mathrm{H}$, overlap, $\mathrm{H} \beta-3), 1.93$ $(1 \mathrm{H}, \mathrm{dd}, J=6.4,3.2 \mathrm{~Hz}, \mathrm{H}-5), 1.82(1 \mathrm{H}$, overlap, $\mathrm{H} \alpha-6$ and $1.76(1 \mathrm{H}$, overlap, $\mathrm{H} \beta-6), 4.05(1 \mathrm{H}, \mathrm{dd}, J=$ 2.8, $2.4 \mathrm{~Hz}, \mathrm{H}-7), 2.99(1 \mathrm{H}, \mathrm{d}, J=14.4 \mathrm{~Hz}, \mathrm{H} \alpha-12)$ and $2.28(1 \mathrm{H}, \mathrm{dd}, J=14.4,2.0 \mathrm{~Hz}, \mathrm{H} \alpha-12), 2.80$ $(1 \mathrm{H}, \mathrm{d}, J=18.0 \mathrm{~Hz}, \mathrm{H} \alpha-14)$ and $2.19(1 \mathrm{H}, \mathrm{dd}, J=18.0,2.0 \mathrm{~Hz}, \mathrm{H} \beta-14), 1.66(1 \mathrm{H}$, sept, $J=6.8 \mathrm{~Hz}, \mathrm{H}-$ 15), $0.93(3 \mathrm{H}, \mathrm{d}, J=6.8 \mathrm{~Hz}, \mathrm{H}-16), 0.94(3 \mathrm{H}, \mathrm{d}, J=6.8 \mathrm{~Hz}, \mathrm{H}-17), 0.94(3 \mathrm{H}, \mathrm{s}, \mathrm{H}-18), 0.89(3 \mathrm{H}, \mathrm{s}, \mathrm{H}-$ 19), 1.18 (3H, s, H-20), $3.98\left(1 \mathrm{H}, \mathrm{d}, J=7.6 \mathrm{~Hz}, \mathrm{H}-1^{\prime}\right), 3.10\left(1 \mathrm{H}, \mathrm{dd}, J=2.8,7.6 \mathrm{~Hz}, \mathrm{H}-2^{\prime}\right), 3.37(1 \mathrm{H}$, dd, $\left.J=2.8,2.4 \mathrm{~Hz}, \mathrm{H}-3^{\prime}\right), 3.43\left(1 \mathrm{H}\right.$, overlapped, H-4'), 3.24 (ddd, $\left.J=5.6,3.2,2.4 \mathrm{~Hz}, \mathrm{H}-5^{\prime}\right), 3.80(1 \mathrm{H}$, $\left.\mathrm{dd}, J=11.6,2.4 \mathrm{~Hz}, \mathrm{H} \alpha-6^{\prime}\right)$ and $3.64\left(1 \mathrm{H}, \mathrm{dd}, J=11.6,5.6 \mathrm{~Hz}, \mathrm{H} \beta-6^{\prime}\right)(\mathrm{S} 1-\mathrm{S} 4$, supplementary materials).

${ }^{13} \mathrm{C}$ NMR $\left(\mathrm{CD}_{3} \mathrm{OD}, 150 \mathrm{MHz}\right.$ ) (Table 1): $\delta 80.91$ (C-3), 25.52 (C-2), 36.19 (C-1), 33.42 (C-4), 40.29 (C-5), 28.59 (C-6), 70.75 (C-7), 151.05 (C-8), 141.44 (C-9), 44.66 (C-10), 204.08 (C-11), 40.13 (C-12), 77.61 (C-13), 52.80 (C-14), 39.54 (C-15), 17.48 (C-16), 16.93 (C-17), 33.63 (C-18), 22.13 (C19), 19.59 (C-20), 105.87 (C-1'), 75.10 (C-2'), 77.71 (C-3'), 71.87 (C-4'), 77.76 (C-5'), 62.86 (C-6') (S5-S10, supplementary materials).

3.2.2. $7 \alpha, 20$-epoxy-1 $\alpha, 6 \beta, 7,14$-tetrahydroxy-kaur-16-en-15-one (oridonin 2):Colorless prism; $[\alpha]_{n}^{20}$ +45.24 (c 0.07, methanol); IR $v_{\max }$ : 3380, 2921, 1711, 1646, $\mathrm{cm}^{-1} ;{ }^{1} \mathrm{H}-\mathrm{NMR}\left(\mathrm{CDCl}_{3}, 400 \mathrm{MHz}\right)$ and ${ }^{13} \mathrm{C}$ NMR $\left(\mathrm{CD}_{3} \mathrm{OD}, 150 \mathrm{MHz}\right.$ ) (Table 2); EIMS $m / z: 364.4[\mathrm{M}]^{+}$; (Calcd for $\mathrm{C}_{20} \mathrm{H}_{28} \mathrm{O}_{6}$ ), data compared with the lietrature [19].

Table 2. ${ }^{1} \mathrm{H}\left(\mathrm{CD}_{3} \mathrm{OD}, 400 \mathrm{MHz}\right),{ }^{13} \mathrm{C} \mathrm{NMR}\left(\mathrm{CD}_{3} \mathrm{OD}, 150 \mathrm{MHZ}\right)$ of Compound 2 (oridonin)

\begin{tabular}{lccccc}
\hline Position & $\boldsymbol{\delta}^{\mathbf{1}} \mathbf{H}$ & $\boldsymbol{\delta}^{\mathbf{1 3}} \mathbf{C}$ & S. No. & $\boldsymbol{\delta}^{\mathbf{1}} \mathbf{H}$ & $\boldsymbol{\delta}^{\mathbf{1 3}} \mathbf{C}$ \\
\hline 1 & $3.72 \mathrm{dd}(6.3,2.3)$ & 65.1 & 11 & $2.01 \mathrm{~m}$ & 19.0 \\
2 & & & & $1.93 \mathrm{~m}$ & \\
3 & $2.40 \mathrm{~m}$ & 27.4 & 12 & $3.92 \mathrm{~d}(4.6)$ & 68.5 \\
4 & $1.57 \mathrm{dd}(6.5,2.4)$ & 40.0 & 13 & $2.03 \mathrm{~m}$ & 38.5 \\
& --- & 34.5 & 14 & $2.4 \mathrm{~m}$ & 31.7 \\
5 & & & & $1.92 \mathrm{~m}$ & \\
6 & $1.80 \mathrm{~d}(6.4)$ & 74.0 & 15 & $1.23 \mathrm{~s}$ & 30.9 \\
7 & $3.7 \mathrm{~d}(6.4)$ & 101.0 & 17 & $4.61 \mathrm{~d}(10.0)$ & 97.1 \\
& --- & 55.0 & 18 & $-24 \mathrm{~s}$ & 15.1 \\
8 & --- & 53.0 & 19 & $6.06 \mathrm{~s}$ & 117.5 \\
9 & $1.84 \mathrm{~d}(5.6)$ & & & $5.38 \mathrm{~s}$ & \\
& & 44.0 & 20 & --- & 199.0 \\
\hline
\end{tabular}

\subsection{Results of the Biological Activities}

The compounds 1 and 2 (Figure 1) were tested for their anticancer, and $\alpha$-glucosidase inhibitory activities which were based on the review of literature reported anticancer, and antihyperglycemic potential of Isodon species.

\subsection{1. $\alpha$-Glucosidase Inhibitory Activity}

Rugosodon 1 (Figure 1) was found with significant $\alpha$-glucosidase inhibition, antihyperglycemic potential with $\mathrm{IC}_{50} 0.453$ while oridonin 2 with $\mathrm{IC}_{50} 0.998 \mathrm{mg} / \mathrm{mL}$, showed comparable activity as compared to the standard acarbose $\left(\mathrm{IC}_{50} 0.921 \mathrm{mg} / \mathrm{mL}\right)$ (Table S3). However, further in vivo testing and optimization of rugosodon $\mathbf{1}$ is required to enhance its potential and structure activity relationship against its $\alpha$-glucosidase target (Table S1, Supplementary materials). 


\subsubsection{Cytotoxic Activity}

The isolated compounds, rugosodon 1 and oridonin $\mathbf{2}$ were evaluated for in vitro growth inhibitory potential against the three human cancer cell lines (NCI-H460, HeLa, and MCF-7) but did not show any significant activities ( $\mathrm{LC}_{50}>50 \mathrm{mg} / \mathrm{ml}$ ) (Table S2, Supplementary materials)

As a conclusion, the results obtained from physicochemical constants and spectroscopic data (IR, ESIMS, EIMS, 1D and 2D NMR) confirm the justification of a new compound named rugosodon (1-O- $\alpha$ - $D$-glucopyranosyl-7 $\alpha, 13 \beta$-dihydroxyabieta-8(9)-en-11one) with significant inhibitory potential of $\alpha$-glucosidase along with a known compound, oridonin isolated from Isodon rugosus Wall Ex Benth.

\section{Acknowledgments}

This research was supported by Ton Duc Thang University, Ho Chi Minh City, Vietnam [Grant \# 881/2016/TDT-HDNCKH]. We are thankful to Abdul Jabar Khan (Charmang College, Bajaur Agency) for providing the plant samples for research work. We are also grateful to the ICCBS, University of Karachi, Pakistan for providing instruments and biological facilities.

\section{Supporting Information}

Supporting information accompanies this paper on http://www.acgpubs.org/journal/records-ofnatural-products

\section{ORCID}

Arif Ullah: 0000-0002-5480-1650

Ghias Uddin: 0000-0003-4501-2972

Mamoon Ur Rashid: 0000-0002-7505-8958

Ismail Ismail: 0000-0002-0037-6097

Nasuriddin Nasuriddin: 0000-0002-9482-4769

Bina Shaheen Siddiqui: 0000-0001-8160-0021

Zahoor Ullah: 0000-0001-5355-1314

Muhammad Alamzeb: 0000-0001-9819-5544

\section{References}

[1] H. D. Sun, S. X. Huang and Q. B. Han (2006). Diterpenoids from Isodon species and their biological activities, Nat. Prod. Rep. 23, 673-98.

[2] S. W. Khan and S. Khatoon (2007). Ethnobotanical studies on useful trees and shrubs of Haramosh and Bugrote valleys In Gilgit Notheren areas of Pakistan, Pak. J. Bot. 39, 699-710.

[3] A. Rashid, M. F. Swati, H. Sher and M. N. Al-Yemeni (2011). Phyto-ecological evaluation with detail floristic appraisal of the vegetation around Malam Jabba, Swat, Pakistan, Asian Pac. J. Trop. Biomed. 1, 461-467.

[4] J. Liu, Q, Wu, J, Shu, R. Zhang and L. Liu (2017). Three new abietane-type diterpene glycosides from the roots of Tripterygium wilfordii, Fitoterapia 120, 126-30.

[5] M. Liu, W. G. Wang, H. D. Sun and J. X. Pu (2017). Diterpenoids from Isodon species: an update, Nat. Prod. Rep. 34, 1090-1140.

[6] A. Abbaskhan, M. I. Choudhary, Y. Tsuda, M. Parvez, F. Shaheen, Z. Parween, R. B. Tareen and M. A. Zaidi (2003). A new diepoxy-ent-kauranoid, Rugosinin, from Isodon rugosus, Planta Med. 69, 94-96.

[7] Z. Sher, Z. Khan and F. Hussain (2011). Ethnobotanical studies of some plants of Chagharzai valley, district Buner, Pakistan, Pak J. Bot. 43, 1445-1452.

[8] N. Akhtar, A. Rashid, W. Murad and E. Bergmeier (2013). Diversity and use of ethno-medicinal plants in the region of Swat, North Pakistan, J. Ethnobiol. Ethnomed. 9, 25-36.

[9] W. Xiang, R. T. Li, Q. S. Song, Z. Na and H. D. Sun (2004). Ent- Clerodanoids from Isodon scoparius, Helv. Chim. Acta. 87, 2860-2865. 
[10] M. Zhou, H. C. Geng, H. B. Zhang, K. Dong, W. G. Wang, X. Du, X. N. Li, F. He, H. B. Qin, Y. Li and J. X. Pu (2012). Scopariusins, A new class of ent-Halimane diterpenoids isolated from Isodon scoparius, and biomimetic synthesis of Scopariusin A and Isoscoparin N. Org. Lett. 15, 314-317.

[11] X. Liu, R. Zhan, W. G. Wang, X. Du, X. N. Li, J. H. Yang, P. Zhang, Y. Li, J. X. Pu, J. Z. Wu JZ and H. D. Sun (2013). New ent-Abietane and ent-Kaurane diterpenoids from Isodon rubescens, Chem. Pharm. Bull. 61, 90-95.

[12] F. Jim-Min, L. Ching-Kuo and C. Yu-Shia (1993). Diterpenes from leaves of Juniperus chinensis, Phytochemistry 33, 1169-1172.

[13] E. Fujita and M. Node (1984). Diterpenoids of Rabdosia species, in: Fortschritte der chemie organischer naturstoffe/progress in the chemistry of organic natural products. Springer. 1, 77-157.

[14] W. Herz, A. Andersen, S. B. Christensen, G. W. Kirby, D. Deepak, R. E. Moore, G. Gäde, A. Khare, U. W. Smit and S. Srivastav (1997). Fortschritte der chemie organischer naturstoffe/progress in the chemistry of organic natural products. Springer Science \& Business Media. 1-106.

[15] H-D. Sun, Z-W. Lin, L-Z. Lin, H-B. Chai, J. M. Pezzuto and G. A. Cordell (1995). Cytotoxic entkaurene diterpenoids from three Isodon species, Phytochemistry 38, 437-442.

[16] B. Y. Hwang, J-H. Lee, T. H. Koo, H. S. Kim, Y. S. Hong, J. S. Ro, K. S. Lee and J. J. Lee (2001). Kaurane diterpenes from Isodon japonicus inhibit nitric oxide and prostaglandin E2 production and NF$\kappa \mathrm{B}$ activation in LPS-stimulated macrophage RAW264. 7 cells, Planta Med. 67, 406-410.

[17] J.-H. Yang, X. Du, F. He, H. -B. Zhang, X-N. Li, J. Su, Y. Li, J. -X. Pu and H-D. Sun. 2013. Bioactive abietane and ent-kaurane diterpenoids from Isodon tenuifolius, J. Nat. Prod. 76, 256-264.

[18] D. M. Piña, C. H. Méndez, L. V. Vargas, H. L. Sunza and J. T. Cortez (2017). Identification and quantification of monosaccharides in Aloe vera gel by acid and enzymatic hydrolysis and wet heat treatment, Food Sci. Nutr. 8, 840.

[19] E. Fujita, T. Fujita, H. Katayama and M. Shibuya (1967). Oridonin, a new diterpenoid from Isodon species, Chem. Commun. 2, 252-254.

[20] N. T. Phat, M. D. Tri, P. N. Minh and B. T. Dat (2015). Two new oleanane-type triterpene saponins from the leaves of Schefflera sessiliflora De PV, Phytochem. Lett. 11, 102-105.

[21] K. Y. Kim, K. A. Nam, H. Kurihara and S. M. Kim (2008). Potent $\alpha$-glucosidase inhibitors purified from the red alga Grateloupia elliptica, Phytochemistry 69, 2820-2825.

[22] B. S. Siddiqui, N. Khatoon, S. Begum, A. D. Farooq, K. Qamar, H. A. Bhatti and S. K. Ali (2012). Flavonoid and cardenolide glycosides and a pentacyclic triterpene from the leaves of Nerium oleander and evaluation of cytotoxicity, Phytochemistry 77, 238-44.

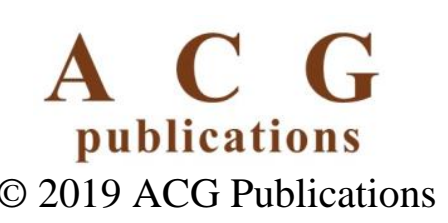

